\title{
Optimisation-based scheduling of an avionic system
}

\section{Emil Karlsson}




\title{
Optimisation-based scheduling of an avionic system
}

\author{
Emil Karlsson
}

11.0U UNNGPDG

Linköping University

Department of Mathematics

Division of Optimization

SE-581 83 Linköping, Sweden

Linköping 2019 
This is a Swedish Licentiate's Thesis

Swedish postgraduate education leads to a doctor's degree and/or a licentiate's degree. A doctor's degree comprises 240 ECTS credits (4 years of full-time studies).

A licentiate's degree comprises 120 ECTS credits.

Edition 1:1

() Emil Karlsson, 2019

ISBN 978-91-7685-056-5

ISSN 0280-7971

URL http://urn.kb.se/resolve?urn=urn:nbn:se:liu:diva-156695

Published articles have been reprinted with permission from the respective copyright holder.

Typeset using $\mathrm{X}_{\mathrm{H}} \mathrm{T}_{\mathrm{E}} \mathrm{X}$

Printed by LiU-Tryck, Linköping 2019 


\section{POPULÄRVETENSKAPLIG SAMMANFATTNING}

I dagens flygplan finns det stora mängder elektronik och funktioner som styrs av datorer, allt ifrån vad som visas på skärmarna för piloten till att flygplanet styrs av pilotens spakar. Detta gör att det är ytterst viktigt att de beräkningar som görs av datorerna inte bara är korrekta utan att de sker i rätt tid, annars kan det få allvarliga följder.

Under de senaste åren har flygplansindustrin gått från att använda federerade datorsystem, där varje dator uppfyller en flygplansfunktion, till integererade modulariserade datorsystem, där flera flygplansfunktioner implementeras på samma dator. Att implementera flera funktioner på samma dator gör det möjligt att minska mängden utrustning i flygplanet och utnyttja utrustningen mer effektivt. En konsekvens av detta är dock att det är mer utmanande att garantera att flygplansfunktionerna inte påverkar varandra. Detta gör att det behövs andra strategier för att garantera att allt genomförs i rätt tid jämfört med om flygplansfunktionerna hade varit implementerade på varsin dator.

I vårt projekt studerar vi ett industriellt relevant problem för framtida flygplan, beskrivet av vår industripartner Saab. För att garantera att allt i datorerna genomförs i rätt tid i detta system behövs ett schema som uppfyller vissa krav. På datorerna körs sedan ett sådant schema vilket tillsammans med andra mekanismer garanterar att allt genomförs $\mathrm{i}$ rätt tid. Arbetet i denna avhandling syftar till att utveckla verktyg för att ta fram sådana scheman eller bevisa att det inte finns ett schema som uppfyller kraven.

Det finns mycket forskning om schemaläggning inom elektroniksystem i flygplan men för att hantera de schemaläggningsproblem som intresserar Saab behövs nya metoder. Detta beror dels på storleken på problemet men också på de tekniska förutsättningarna. Schemaläggningsverktyget som vi har utvecklat är baserat på metoder för att lösa diskreta optimeringsproblem. Strategin som vi har tagit fram är baserad på bivillkorsgenerering, vilket är en metod som tar bort vissa krav och på ett systematiskt sätt återinför dem om de behövs. Metoden som vi presenterar innehåller också komponenter som gör att sökningen på ett systematiskt sätt fokuserar på olika delar av problemet. Med denna metod kan vårt schemaläggningsverktyg lösa problem med över 45000 aktiviteter. 


\begin{abstract}
Modern computer systems in aircraft are often based on an integrated modular avionic architecture. In this architecture, software applications share hardware resources on a common avionic platform. Many functions in an aircraft are controlled by software and a failure in such software can have severe consequences. In order to avoid malfunction, there are many aspects to consider. One aspect is to ensure that the activities in the system is done at the right time with the right resources. To analyse if this is possible or not is often called schedulability analysis.
\end{abstract}

When multiple functions are using the same resources, the schedulability analysis becomes increasingly challenging. This thesis focuses on a pre-runtime scheduling problem of an integrated modular avionic system proposed by our industrial partner Saab. The purpose of this problem is to find a feasible schedule or prove that none exists as part of a schedulability analysis.

For the system that we study, there are two major challenges. One is that task and communication scheduling are integrated and the other is that there is a large amount of tasks to schedule. For the largest instances, there are more than 10000 tasks on a single module. In order to solve such problems, we have developed a matheuristic. At the core of this matheuristic is a constraint generation procedure designed to handle the challenges of the scheduling problem.

The constraint generation procedure is based on first making a relaxed scheduling decision and then evaluating this in a separate problem where a complete schedule is produced. This yields a decomposition where most technical details are considered in the relaxed problem, and the actual scheduling of tasks is handled in a subproblem. Both the relaxed problem and the subproblem are formulated and solved as mixed integer programs.

The heuristic component of the matheuristic is that the relaxed problem is solved using an adaptive large neighbourhood search method. Instead of solving the relaxed problem as a single mixed integer program, the adaptive large neighbourhood search explores neighbourhoods through solving a series of mixed integer programs. Features of this search method are that it is made over both discrete and continuous variables and it needs to balance feasibility against profitable objective value.

The matheuristic described in this thesis has been implemented in a scheduling tool. This scheduling tool has been applied to instances provided by our industrial partner and to a set of public instances that we have developed. With this tool, we have solved instances with more than 45000 tasks. 


\section{Acknowledgements}

First I would like to give thanks to my supervisor Elina Rönnberg. You have always taken time to help me during this endeavour when I have had questions or needed to discuss anything. This is something I truly appreciate. I also want to thank my supervisors Kaj Holmberg and Simin Nadjm-Tehrani. I appreciate the time you have taken to help me during this time. I also want to thank my colleagues at the Department of Mathematics for good discussions, help with administration, and providing a good place to work.

To do the thesis in the way it has been done would have not been possible without Saab. Thanks to all people who has been involved in making this possible. Particular thanks to Andreas Stenberg and Hannes Uppman, who I have worked closely with during this time and learned a lot from and together with. It has been many good and interesting discussions, whether it has been software, research, or anything else.

I want to thank the Research school in Interdisciplinary Mathematics for funding my research and granting me the possibility to learn about optimisation and real-time systems.

At last I would like to the people closest to me. Thank you Tahere for always being so kind and giving the best advice about life. Thank you Ghazaleh, the love of my life, if I hadn't met you my life would have not been so good, I love you always.

Emil Karlsson 


\section{Contents}

Sammanfattning iii

Abstract iv

Acknowledgments v

Contents vi

List of Abbrevations $\quad$ ix

1 Introduction $\quad 1$

1.1 Outline of the thesis summary . . . . . . . . . . . . . . 2

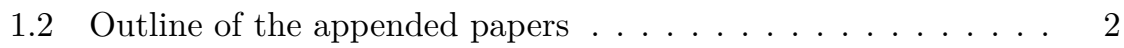

1.3 Presentations ....................... 4

2 Avionic scheduling $\mathbf{5}$

2.1 The development of avionic architectures . . . . . . . . . 6

2.2 An integrated modular avionic scheduling problem . . . . . . . 8

3 Single machine scheduling and mixed integer programming 13

3.1 A brief historical perspective . . . . . . . . . . . . . 13

3.2 Single machine scheduling with release times and deadlines . . 14

3.3 Time-based formulations . . . . . . . . . . . . . . . . . . . . . . . . . . 14

3.4 Order-based formulations . . . . . . . . . . . . . . . . . . . . . . . . . . .

3.5 Position-based formulation . . . . . . . . . . . . . . 20

3.6 Comparisons of mixed integer programming formulations . . . . 21

4 Contributions $\mathbf{2 5}$

4.1 A large-scale problem in avionic scheduling . . . . . . . . . . . . 25

4.2 A solution approach for avionic scheduling . . . . . . . . . . 27

4.3 An exact solution approach for single machine scheduling . . . 29

4.4 A matheuristic for avionic scheduling . . . . . . . . . . . . . . 32

$\begin{array}{ll}\text { Bibliography } & 35\end{array}$ 
Paper A

Paper B

71

Paper C

79 



\section{List of Abbrevations}

AFDX - Avionics Full-DupleX Switched Ethernet

ALNS - Adaptive large neighbourhood search

ARINC - Aeronautical Radio, Incorporated

ASHLEY - Avionics systems hosted on a distributed modular electronics large scale demonstrator for multiple types of aircraft

AM - Application module

CM - Communication module

CN - Communication network

EASA - European Aviation Safety Agency

FAA - Federal Aviation Administration

IMA - Integrated modular avionics

MIP - Mixed integer programming

NASA - National Aeronautics and Space Administration

RTCA - Radio Technical Commission for Aeronautics

SCARLETT - Scalable \& reconfigurable electronics platforms and tools

TDMA - Time-division multiple access

TSP - Travelling Salesman Problem

TTEthernet - Time-Triggered Ethernet 



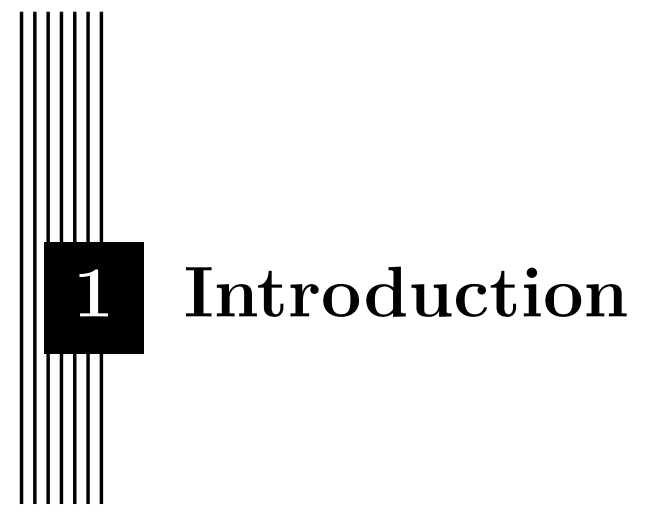

The overall purpose of this research project is to study scheduling and resource allocation problems in real-time systems. This thesis contributes to this area by developing solution techniques based on optimisation for a scheduling problem. The scheduling problem was proposed by our industrial partner, the Swedish defence and security company Saab, and it is of interest in the development of future avionic systems. This thesis consists of a thesis summary and three appended papers. We start by introducing the application area of this thesis, which is real-time systems or, more specifically, avionic systems.

Modern aircraft contain a lot of electronics, such as sensors, actuators, buses, and computers, that respond to, control, and measure the state of the aircraft. The electronics of an aircraft is called avionics or the avionic system of an aircraft. Much of the basic functionality of the aircraft, such as steering, navigation, and communication, relies on the avionic system behaving properly. However, the proper behaviour might be different depending on the actions of the pilot, the position of the aircraft, or the physical time. Systems of this type, where the proper behaviour does not only depend on the results of calculations, but also when they are performed, are examples of real-time systems.

In order to avoid malfunction in a real-time system, it is of vital importance to make sure that the components of the system do the correct thing at the correct time with sufficient resources (computing time, data, et cetera). Research on scheduling of real-time systems makes an effort to analyse these 
situations from a scheduling perspective, and can, depending on the context, be applied during different stages of the development of the system.

The following two sections describe the thesis summary along with the appended papers and their publication status, while the last section lists presentations of this material at conferences.

\subsection{Outline of the thesis summary}

The contributions of this thesis are in two research areas: avionic scheduling and scheduling techniques based on optimisation. The thesis summary treats relevant aspects from these areas in four chapters. The objective of this is to put the research of the appended papers in perspective. Below, the four chapters in the thesis summary are described.

This first chapter gives a general introduction and describes the structure of this thesis. Chapter 2 gives an introduction to scheduling of avionic systems. That chapter has been written for a reader with a background in optimisation and it ends with a description of the avionic scheduling problem that is the optimisation problem studied in this thesis.

In Chapter 3, mixed integer programming (MIP) formulations for single machine scheduling are reviewed. Single machine scheduling is a core problem structure of the avionic scheduling problem treated in this thesis. The last chapter of the thesis summary, Chapter 4, summarises the contributions of the appended papers.

\subsection{Outline of the appended papers}

There are three appended papers in this thesis, and they are included in chronological order. This section gives a short description of each of them along with their publication status and an author statement.

\section{Paper A - An optimisation approach for pre-runtime scheduling of tasks and communication in an integrated modular avionic system}

Paper A is published in the journal Optimization and Engineering, volume 19, issue 4 in pages $977-1004,2018$.

Authors: Mathias Blikstad, Tomas Lööw, Emil Karlsson and Elina Rönnberg

Abstract: The first paper introduces an industrially relevant pre-runtime avionic scheduling problem along with a suitable exact solution approach. The solution approach relies on constraint generation. This procedure is im- 
plemented in a scheduling tool that is tested on three instances provided by Saab.

Author statement: I have contributed to the modelling, the MIP formulation, the implementation, the testing, and to writing the paper.

\title{
Paper B - Explicit modelling of multiple intervals in a constraint generation procedure for multiprocessor scheduling
}

Paper B is published in pages 567-572 of the peer-reviewed conference proceeding, Operations Research Proceedings 2017, by Springer International Publishing. Editors: N. Kliewer, J.F. Ehmke, and R. Borndörfer.

Authors: Emil Karlsson and Elina Rönnberg

\begin{abstract}
Paper B improves the exact constraint generation procedure presented in Paper A by the introduction of sub-intervals. The sub-intervals enable a stronger relaxation in the constraint generation procedure and a smoother connection to other technical requirements.
\end{abstract}

Author statement: I have been responsible for the implementation and testing for this paper. The modelling and writing have been done together with the supervisor.

\section{Paper C - A matheuristic approach to large-scale avionic scheduling}

Paper $\mathrm{C}$ has been partially presented at the conference Practice and Theory of Automated Timetabling 2018 (PATAT 2018) based on a peer-reviewed extended abstract. The full version of Paper $\mathrm{C}$ has been submitted to a journal, and has been published as an MAI-report, ISSN 0348-2960, with number LiTH-MAT-R-2019/02-SE, 2019.

Authors: Emil Karlsson, Elina Rönnberg, Andreas Stenberg and Hannes Uppman

\begin{abstract}
The third paper extends the exact constraint generation procedure presented in Paper A into an matheuristic. In the matheuristic, the relaxed problem is solved using an adaptive large neighbourhood search (ALNS) that explore neighbourhoods using a MIP solver. This work also presents improved MIP formulations for models in the constraint generation procedure, which effect both the exact approach and the matheuristic. The exact approach and the matheuristic are evaluated on 5 Saab instances and 120 public instances.
\end{abstract}


The results indicate that the matheuristic performs better than the exact approach on large instances.

Author statement: I have contributed to the modelling, the MIP formulations and the solution approaches of this paper, and have, under supervision, done a large part of the writing. I have also been responsible for creating the public instances and have been involved in the implementation and testing.

\subsection{Presentations}

During my doctoral studies I have attended and made presentations at the following conferences.

- International Conference on Operations Research 2017 (OR2017). This conference took place in Berlin, Germany, in September 2017. I presented the work of Paper B.

- Svenska operationsanalyskonferensen 2017 (SOAK2017). This conference took place in Linköping, Sweden, in October 2017. I presented the work of Paper B. 


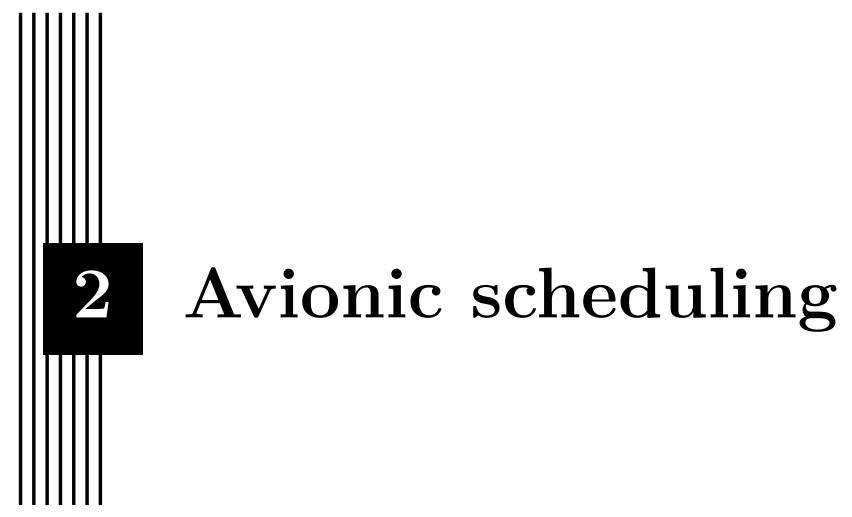

This section gives a brief introduction to the technical application area of this thesis, that is, scheduling of avionic systems. The introduction is aimed to give a person with a background in optimisation sufficient information to understand the motivation for the problem studied in this thesis.

Computer systems where the correct behaviour is a function of time and does not only depend on the result of a computation are called real-time computer systems. The combination of such a computer system together with its interaction, for example a human through a keyboard, or a human interface, is called a real-time system.

Real-time computer systems can be classified into hard real-time systems or soft real-time systems. A hard real-time system is a system where a single missed deadline (the instant a result must be produced) could have severe consequences. Examples of hard real-time systems are components in traffic lights at an intersection, the steering of an aircraft, the braking system in a car, the control process of a nuclear plant, and launching a rocket into space. A soft real-time system is a system where a missed deadline does not cause severe consequences. Examples of soft real-time systems could be streaming a movie on your computer or calling on a cell-phone. The work in this thesis is concerned with hard real-time systems in aircraft.

In order to avoid a missed deadline in a hard real-time system, each task must be given enough resources and be performed at the right time. The analysis that answer if this is possible or not is often called schedulability 
analysis. Schedulability analysis can be done in many different ways, and in different stages of the development process of a system.

Problems associated with schedulability analysis at an early stage of the development process are often related to resource allocation, for example if it is possible to allocate tasks to modules and satisfy certain constraints. However, in the later stages of the development process the problems are often related to scheduling, for example if a schedule that satisfy constraints exists or if a scheduling algorithm will work under certain task loads.

An important distinction for scheduling problems in real-time systems are whether or not the analysis is done at runtime or before runtime (called preruntime). In runtime scheduling the task load is typically not known and a scheduling algorithm might guarantee a working system under certain task loads. In pre-runtime scheduling the entire task load is known beforehand and thus a scheduling algorithm using this or a schedule that satisfies these requirements can be produced.

The problem studied in this thesis belongs to the later stages of the development process and is about producing a pre-runtime schedule. The following sections give a description of the development of avionic architectures and the scheduling problem studied in this thesis. For further information about scheduling in real-time systems and avionic systems, see the introductory parts of PhD-theses [35] and [33], respectively.

\subsection{The development of avionic architectures}

An aircraft is often developed and maintained through many years (even decades) and receives regular updates of software and hardware during its lifetime. In order to create a safe and reliable aircraft there are many different regulations and guidelines regarding the components in an aircraft. For example, there are guidelines for the software development and verification process of software in an aircraft, the most recent being DO-178C by Radio Technical Commission for Aeronautics (RTCA), see [29].

Since an aircraft is supported through many years, updates of software and hardware might be necessary due to advances since the introduction of the aircraft. Thus, an avionic architecture that support incremental development and verification of software and hardware is something to strive for. Agencies, such as European Aviation Safety Agency (EASA) in Europe and Federal Aviation Administration (FAA) in the USA, provide guidelines on how to ease incremental certification of an avionic system.

When computers first were used in aircraft, each avionic function with electronic components, such as steering, navigation, et cetera, was implemented on a dedicated computer connected to the sensors and actuators it required. If different avionic functions needed to interact with each other, direct cables 
were drawn between them. This kind of avionic system is referred to as a federated system.

The federated systems underwent several transitions. In the beginning, avionic systems used analogue circuits. Later digital circuits and more sophisticated processors were introduced. As part of this transition, there has been different methods to transfer data within an avionic system. For example, Aeronautical Radio, Incorporated (ARINC) 429 and MIL-STD-1553 published by the United States Department of Defense.

As the amount of avionic functions that were controlled by software grew, so did the size and weight of the federated systems. Beginning in the late 1980s and early 1990s, a new architecture of avionic systems emerged, called integrated modular avionics (IMA) systems. In this setting, multiple avionic functions could be implemented on the same hardware, utilising the computing resources more efficiently. Years later, guidelines on how certify such modules were agreed upon, see DO-297 by RTCA [30]. During these years, different guidelines and Ethernet networks were introduced to improve data transfer in avionic systems. Examples of these are ARINC 664 Ethernet network, Avionics Full-DupleX Switched Ethernet (AFDX) developed by Airbus, and Time-Triggered Ethernet (TTEthernet).

During the last two decades, there has been multiple large research efforts that investigate more advanced avionic architectures. Examples of such research efforts are the National Aeronautics and Space Administration (NASA) Orion Space program in the USA, that investigated the avionic architecture for future space travels, and the Scalable \& reconfigurabLe electronics platforms and tools (SCARLETT) project and the Avionics systems hosted on a distributed modular electronics large scale demonstrator for multiple types of aircraft (ASHLEY) project in Europe, that examined the next generation of IMA systems.

An avionic architecture that was further distributed was suggested as part of this research, for example see [13]. In such a system, different IMA units are located in the same avionic system, but they are connected through an Ethernet network. Such an architecture would have the advantages of the IMA system, such as better resource utilisation, while allowing for physical disjoint subsystems. Key challenges for distributed IMA systems are reviewed in [37], where system schedulability analysis is one of the challenges.

A disadvantage of creating a complex architecture is that, as the system architecture grows increasingly complex, so does the challenge of analysing them. When multiple avionic functions are implemented on the same hardware, you need to ensure that all software applications that implement the avionic functions get sufficient computing resources without affecting any other software application. This increases the complexity in design and verification with respect to things such as fault containment and scheduling. ARINC 653 provide an example of guidelines on how to handle this type of partitioning. 
This thesis focuses on developing solution methods for a scheduling problem of interest in the development of future IMA systems. The objective of the scheduling problem is to produce a global pre-runtime schedule for both the processors and the Ethernet network of a particular IMA system. Details of this problem and its avionic system is found in Section 2.2.

\subsection{An integrated modular avionic scheduling problem}

The following section introduces the avionic scheduling problem described in Paper C. This scheduling problem is of interest in the development of future IMA systems and has been proposed by our industrial partner Saab. Paper A and Paper B studied a restriction of this problem where some modelling simplifications were made. This difference is further described in Section 2.2.

The problem is to construct a feasible schedule or prove that no schedule exists. In this system, a schedule shall be produced before runtime in a setting where tasks, worst case execution times, and requirements of the system are known beforehand. The system is periodic and the schedule repeats once every major period (major frame). Typically, the length of a major period is about one second with a time resolution in nanoseconds.

In the system, there are a set of nodes comprised of different types of modules (processors) that performs tasks. Every task in the system must be performed on a specific module and is not allowed to migrate. Each node has a single communication module $(\mathrm{CM})$ and one or more application modules (AMs). The CMs are connected to a single communication network $(\mathrm{CN})$, allowing the nodes to communicate with another by sending messages. The system is illustrated in Figure 2.1. The decisions required to create a schedule are assigning start times to tasks and assigning send times to messages on the $\mathrm{CN}$.

The purpose of the AMs is to host software applications. The software applications are grouped into partitions by engineers. The partitions are then scheduled on the AMs as tasks. On an AM, there is often few tasks with long execution requirements. The CMs are responsible for the communication of a node. This includes internal communication between the modules and external communication through the $\mathrm{CN}$ or with other external equipment. A CM often includes many tasks, with short execution requirements.

\section{Module scheduling}

Each task in the system is repeated at least once every major period. The quotient of the major period and the period of a task determine the number of times this task is performed in a major period. The period of a task always divides the major period, and on a particular module the task periods are all the same. Tasks on an AM are performed 64 times during a major period, while on the CMs, they are performed one time during a major period. To 


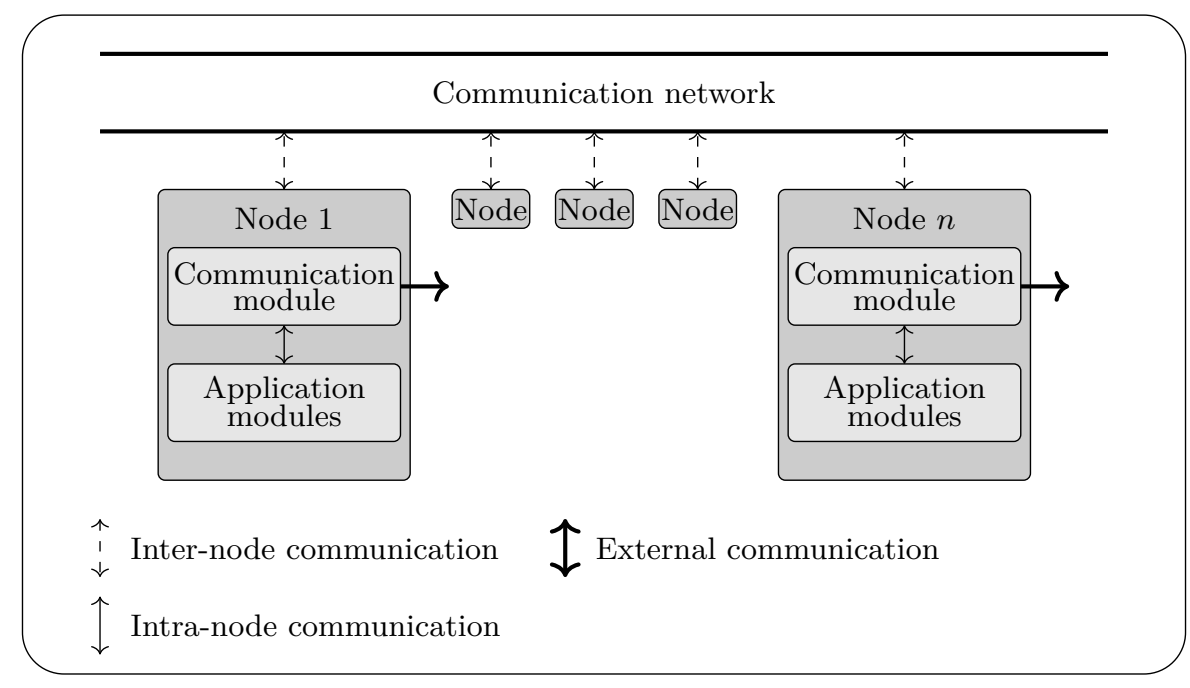

Figure 2.1: An overview of a system that consist of $n$ nodes. The structure of the content is the same for all nodes and therefore only displayed for node 1 and $n$

uniquely identify an occurrence of a task in a major period we introduce task instances. A task instance is a tuple of an integer and a task identifier that uniquely identifies a particular occurrence of a task in a major period.

A module can only perform a single task at a given time, and no pair of tasks on the same module are allowed to overlap. Thus, each task must be granted a non-preempted time slot with the duration of its execution requirement on its module. The execution requirement of a task corresponds to its worst-case execution time or its worst-case duration. An additional requirement is that the non-preempted time slot of each task must be within one of its given sub-intervals, where a sub-interval consists of a release time and a deadline pair.

\section{Precedence relations}

There are two types of precedence relation constraints in the problem, referred to as dependencies and chains. The precedence constraints ensure that data are processed in the correct order within the system. The precedence constraints called dependencies, restrict the duration between a pair of tasks, where the tasks can be on the same or on different modules. Formally, a dependency restricts the duration from the start of a task instance to the start of another a task instance to be greater than or equal to a minimum length and smaller than or equal to a maximum length. 
The second type of precedence relations constraints, the chains, restrict the duration between the start and end of a sequence of tasks, where the tasks can be on the same or on different modules. Formally, a chain restricts the duration from the start to the end of a cyclic order of task instances to be less than a major period. Adjacent task instances in a chain are often connected via dependencies.

\section{Communication network}

The role of the $\mathrm{CN}$ is to send messages between the CMs in the system. In the $\mathrm{CN}$, there exists a set of pre-defined slots every major period where messages can be sent. Each message on the $\mathrm{CN}$ is sent from a single CM to a set of receiving $\mathrm{CMs}$ and must be allocated to a slot. In order to send and receive a message, four types of tasks are involved. On the sending CM, there are two tasks responsible for preparing and sending the message, while on each receiving $\mathrm{CM}$, there are two tasks that dequeue the message and read the data. The four types of tasks involved in sending and receiving a message must be performed in a particular order, which is enforced by dependencies and chains.

Each message requires a certain bandwidth in the slot it is sent in. Thus, the required capacity of the messages sent in a slot is not allowed to exceed the bandwidth of that slot. Two additional constraints are that tasks must be dequeued in the order that the messages are sent, and that the tasks responsible for sending and dequeuing the message must be performed within certain sub-intervals, where the sub-intervals depend on the slot their message has been assigned to.

If two or more messages are sent in the same slot, their tasks of the same type must be performed in immediate succession if they are performed on the same CM. This is referred to as co-allocation of messages in a slot. A benefit of co-allocation of messages, is that the tasks that must be performed in immediate succession get reduced execution requirements compared to if they were performed separately. In Paper A and Paper B, no co-allocation of messages in a slot is allowed.

\section{Technical orientation of the system}

This section provides some additional comments with respect to technical details. The purpose of this is to put the scheduling problem into perspective. The IMA system we address is synchronous. The purpose of the pre-runtime scheduling is to create a global static schedule for this system. The system is then run by dispatchers according to this global static schedule. The only case of runtime scheduling of the system is done inside the partitions on the AMs where the software applications are run by a rate-monotonic scheduler. The CN that we describe is a time-division multiple access (TDMA) Ether- 
net network that supports multicast, see patent [10]. For further technical orientation and related research of this problem, see the introductory parts of Paper A and Paper C. 



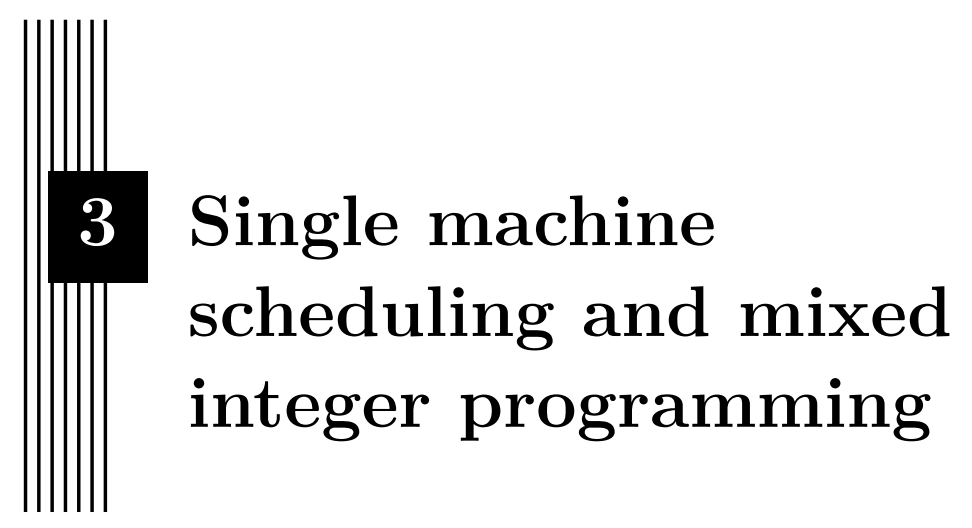

A core aspect of many large-scale scheduling problems is to create a schedule for one machine. The most fundamental of such scheduling problems are the single machine scheduling problems. In a single machine scheduling problem, a set of tasks (jobs or activities) shall be scheduled without overlap on a single processor (machine or resource).

Since scheduling of machines often is a core part of a larger scheduling problem, the performance of such scheduling is critical to obtain overall computational efficiency. As such, single machine scheduling problems have been extensively studied. In this section, we survey MIP formulations for a single machine scheduling problem with release times and deadlines, where the purpose is to find out if a feasible schedule exists or not.

\subsection{A brief historical perspective}

Shortly after the introduction of MIP by [15], three MIP approaches to solve scheduling problems were introduced. The approaches differed in their choice of binary decision variable. The three main types of formulations were: in [8] the variable was time-based by assigning each task a start time, in [36] the variable was position-based by assigning each task a position in the schedule, and in [23] the variable was order-based by assigning an order between each pair of tasks.

Studying the polyhedron for an order-based decision variable, [3] began a series of polyhedral results of different MIP formulations for single machine 
scheduling. The three main categories were studied in different papers with slightly different problem formulations, see details in the following sections. In [28], these recent advances in polyhedral results for single machine scheduling were summarised.

During the last two decades, there has been some theoretical advances, primarily in the position-based approach, see [20] and [11]. There have also been some new extensions with a time-based approach, see [4] and [7]. But there have also been some computational comparisons evaluating the performance on different formulations, see [4], [19], and [2].

\subsection{Single machine scheduling with release times and deadlines}

In this section, we introduce the terminology and notation that will be used for non-preemptive single machine scheduling with release times and deadlines in the following sections. Let $\mathcal{I}$ denote a set of tasks that shall be scheduled without pre-emption on a single machine that can handle at most one task at a time. Associated with each task $i \in \mathcal{I}$, is a release time $r_{i}$ and a deadline $d_{i}$, specifying an interval $\left[r_{i}, d_{i}\right]$ wherein the task must be processed. The processing time (or duration) of task $i \in \mathcal{I}$ is denoted by $p_{i}$. The aim is to evaluate if there exist a feasible schedule (and if one such exists; give it) that respect the release time and deadline of each task. Using the $\alpha|\beta| \gamma$ classification scheme in [22], a slight extension of the one originally introduced in [16], this problem can is classified as $1\left|r_{j}, \bar{d}_{j}\right|-$. In [14], this problem was proven to be NP-complete for arbitrary release times, deadlines and processing times.

\subsection{Time-based formulations}

In this section, we present different formulations centred on time-based binary decision variables. Since the number of variables depends on the scheduling horizon, for a problem with long scheduling horizon the time-indexed approach is not practical in every circumstance. Therefore, some new formulations with this in mind, such as the interval-indexed formulation and the bucket-indexed formulation are presented.

\section{Time-indexed formulation}

The time-indexed approach divides the scheduling horizon $T=\max _{i \in \mathcal{I}} d_{i}$ into unit size time periods and uses a binary decision variable to state whether a particular task start at a particular time period. This requires that all processing times of tasks are integer (or at least rational), since the resolution will be the unit sized time period. 
Introduce for task $i \in \mathcal{I}$, and time period $t, t=0, \ldots, T$, the binary variable

$$
x_{i t}= \begin{cases}1, & \text { if task } i \text { starts in time period } t \\ 0, & \text { otherwise }\end{cases}
$$

By finding a solution that obey

$$
\begin{gathered}
\sum_{t^{\prime}=0}^{T-p_{i}+1} x_{i t^{\prime}}=1, \quad i \in \mathcal{I}, \\
\sum_{i \in \mathcal{I}} \sum_{t^{\prime}=t-p_{i}+1}^{t} x_{i t^{\prime}} \leq 1, \quad t=0, \ldots, T, \\
\sum_{t^{\prime}=0}^{r_{i}-1} x_{i t^{\prime}}=0, \quad i \in \mathcal{I}, \\
\sum_{t^{\prime}=d_{i}-p_{i}+1}^{T} x_{i t^{\prime}}=0, \quad i \in \mathcal{I}, \\
x_{i t} \in\{0,1\}, \quad t=0, \ldots, T, \quad i \in \mathcal{I},
\end{gathered}
$$

we have a feasible schedule for the single machine scheduling problem with release times and deadlines. In the formulation, Constraint (3.2) assigns each task a time period, Constraint (3.3) prevents execution of more than one task in a time period, Constraint (3.4) makes sure that each task respect its release date, while Constraint (3.4) makes sure that each task respect its deadline. Observe that Constraints (3.4)-(3.5) could be replaced by setting $x_{i t}=0$ for each task $i$ in time period $t \notin\left\{r_{i}, \ldots, d_{i}\right\}$.

The time-indexed formulation to a scheduling problem was introduced by [8], for what we today would call a machine scheduling problem. In this initial formulation the binary variable used was slightly different, instead indicating whether or not a task should be performed at a given time. Some years later, [26] presented a variation of this variable definition with a binary variable that indicates if a task finish at a certain time period. In the early 1990s theoretical properties and inequalities for the time-indexed formulation were studied by [12]. In [34], further valid inequalities based on knapsack relaxations were presented along with a procedure to obtain such valid inequalities. This procedure was used in a cutting plane framework that was tested on single machine scheduling instances. More results were published in [1], extending the results of [12], focusing on inequalities with right-hand side equal to 1 and 2 .

\section{Interval-indexed formulation}

The interval-indexed formulation was introduced in [4]. In this formulation, the scheduling horizon is partitioned into intervals. Let $\mathcal{Q}$ denote the set of intervals. The lengths of the intervals are arbitrary and for interval $q \in \mathcal{Q}$, the time-points $\left(t_{q}^{\mathrm{S}}, t_{q+1}^{\mathrm{S}}\right]$ define the interval. For each interval $q \in \mathcal{Q}$, let $\mathcal{I}_{q}^{\mathrm{S}}$ 
denote the set of tasks with a processing time less than or equal to the length of the interval. Similarly, let $\mathcal{I}_{q}^{\mathrm{B}}$ denote the set of tasks with a processing time larger than the length of the interval $q \in \mathcal{Q}$.

The interval-indexed formulation is based on two decisions: determine an interval for each task to start in and measure the idle time for each interval. In order to create a valid formulation, the authors prove that the tasks can always be ordered in a specific way that makes the idle time for an interval well defined.

For each task $i \in \mathcal{I}$ and interval $q \in \mathcal{Q}$, introduce the binary variables

$$
z_{i q}^{\mathrm{S}}= \begin{cases}1, & \text { if task } i \text { starts in interval } q \text { or earlier } \\ 0, & \text { otherwise }\end{cases}
$$

and

$$
z_{i q}^{\mathrm{E}}= \begin{cases}1, & \text { if task } i \text { is completed in interval } q \text { or earlier } \\ 0, & \text { otherwise. }\end{cases}
$$

Also introduce for each interval $q \in \mathcal{Q}$, the continuous variable

$$
u_{q}^{\text {idle }}=\text { the idle time of interval } q .
$$

With these variables, the single machine scheduling problem with release times and deadlines can be formulated as

$$
\begin{gathered}
z_{i q}^{\mathrm{E}} \leq z_{i, q+1}^{\mathrm{E}}, \quad q \in \mathcal{Q}, i \in \mathcal{I}, \\
z_{i q}^{\mathrm{S}} \leq z_{i, q+1}^{\mathrm{S}}, \quad q \in \mathcal{Q}, i \in \mathcal{I}, \\
z_{i q}^{\mathrm{E}} \leq z_{i q}^{\mathrm{S}}, \quad q \in \mathcal{Q}, i \in \mathcal{I}, \\
z_{i q}^{\mathrm{S}} \leq z_{i, q+1}^{\mathrm{E}}, \quad i \in \mathcal{I}_{q}^{\mathrm{S}}, \quad q \in \mathcal{Q}, \\
z_{i q}^{\mathrm{E}} \leq z_{i, q-1}^{\mathrm{S}}, \quad i \in \mathcal{I}_{q}^{\mathrm{B}}, \quad q \in \mathcal{Q}, \\
\sum_{i \in \mathcal{I}} p_{i} z_{i q}^{\mathrm{E}}+\sum_{1}^{q} u_{q}^{\mathrm{idlle}} \leq t_{q+1}^{\mathrm{S}}, \quad q \in \mathcal{Q}, \\
\sum_{i \in \mathcal{I}} p_{i} z_{i q}^{\mathrm{S}}+\sum_{1}^{q} u_{q}^{\mathrm{idle}} \geq t_{q+1}^{\mathrm{S}}, \quad q \in \mathcal{Q}, \\
u_{q}^{\mathrm{idle}} \leq\left(t_{q+1}^{\mathrm{S}}-t_{q-1}^{\mathrm{E}}\right)\left(1-\sum_{i \in \mathcal{I}}\right)\left(z_{i q}^{\mathrm{S}}-z_{i, q+1}^{\mathrm{E}}\right), \quad q \in \mathcal{Q}, \\
\sum_{i \in \mathcal{I}_{q}^{\mathrm{B}}}\left(z_{i q}^{\mathrm{S}}-z_{i, q+1}^{\mathrm{E}}\right)+z_{i^{\prime}, q+1}^{\mathrm{E}}-z_{i^{\prime} q}^{\mathrm{S}}, \quad i^{\prime} \in \mathcal{I}_{q}^{\mathrm{S}}, \quad q \in \mathcal{Q} .
\end{gathered}
$$


Constraints (3.7) and (3.8), ensure consistent definition of the binary variables. Constraint (3.9) makes sure that if a task is completed in some interval, it should be started in this interval or earlier. Constraint (3.10) forces the fact that, if a task does not fit inside an interval, it cannot start before this interval and end in an later interval. Constraint (3.11) ensures that tasks that are larger than an interval cannot start and end in the same interval. Constraints (3.12) and (3.13), ensure that the processing times of task scheduled up to the start of an interval with the idle time is consistent with the time spent. Constraint (3.14) puts idle times to zero if a task starts before the interval and ends after the interval. Constraint (3.15) eliminates overlap between a task that is too large for an interval and a task that is fully processed within that interval. Put the corresponding $z_{i q}^{\mathrm{S}}$ and $z_{i q}^{\mathrm{E}}$ equal to zero to account for release times and deadlines. For a proof that this will yield a feasible schedule, see [4].

A formulation similar to the interval-indexed formulation is used in [9]. In [9], a logic-based Benders decomposition scheme is used to solve a single machine scheduling problem. The scheme is to, assign an interval to each task in a relaxed problem and evaluate an assignment of intervals to tasks in a subproblem. The authors solve the relaxed problems using a MIP solver while the subproblems are solved using a constraint programming solver. This approach is applied to two single machine scheduling problems. One is the problem we describe in Section 3.2 and the other is a single machine problem where intervals are disjoint, meaning that each task must be fully performed within an interval.

\section{Bucket-indexed formulation}

The bucket-indexed formulation was suggested by [7] and was inspired by similar approaches used in lot-sizing. In this formulation, the scheduling horizon is divided into buckets of equal size. However, the bucket size $p_{B}$ is selected to be less than the shortest processing time of all tasks, hence $p_{B} \leq \min _{i \in \mathcal{I}} p_{i}$. The reason for this is to make sure that only a single task can start in a bucket, and each task must span at least two buckets. The bucket-indexed approach is similar to the idea of the time-indexed formulation, which can be seen as a special case of the bucket formulation where the bucket size is equal to one.

Let $\{1, \ldots, B\}$ denote the buckets. For each task $i \in \mathcal{I}$, let $B_{i}$ denote the number of buckets it must at least span. For each task $i \in \mathcal{I}, k \in\{0,1\}$, and bucket $b \in\{1, \ldots, B\}$, introduce the binary variable

$$
z_{i b k}= \begin{cases}1, & \text { if task } i \text { starts in bucket } b \text { and finishes in bucket } b+B_{i}+k, \\ 0, & \text { otherwise. }\end{cases}
$$


For each task $i \in \mathcal{I}$, introduce the continuous variable

$\pi_{i}=$ fraction of the bucket task $i$ ends in after the processing of task $i$.

Also, for each task $i \in \mathcal{I}, k \in\{0,1\}$, and bucket $b \in\{1, \ldots, B\}$, introduce the continuous variables

$$
u_{i b k}^{\mathrm{S}}=\left\{\begin{array}{l}
\text { fraction of task } i \text { spent in bucket } b \text { if task } i \text { starts in bucket } b \\
\text { and spans } B_{i}+k \text { buckets, }
\end{array}\right.
$$

and

$$
u_{i b k}^{\mathrm{E}}=\left\{\begin{array}{l}
\text { fraction of task } i \text { spent in bucket } b \text { if task } i \text { ends in bucket } b \\
\text { and spanned } B_{i}+k \text { buckets. }
\end{array}\right.
$$

The bucket-indexed formulation can then be formulated as

$$
\begin{gathered}
\sum_{k \in\{0,1\}} \sum_{b \in\{1, \ldots, B\}} z_{i b k}=1, \quad i \in \mathcal{I}, \\
\sum_{i \in \mathcal{I}} \sum_{k \in\{0,1\}} \sum_{b \in\left\{b^{\prime}-B_{i}-k+2, \ldots, b^{\prime}\right\}} z_{i b k} \leq 1, \quad b^{\prime} \in\{1, \ldots, B\}, \\
\sum_{i \in \mathcal{I}} \sum_{k \in\{0,1\}}\left(u_{i b k}^{\mathrm{S}}+u_{i b k}^{\mathrm{E}}+\sum_{b \in\left\{b^{\prime}-B_{i}-k+2, \ldots, b^{\prime}-1\right\}} z_{i b k}\right) \leq 1, \quad b^{\prime} \in\{1, \ldots, B\}, \\
u_{i b k}^{\mathrm{S}}+u_{i, b+B_{i}+k-1, k}^{\mathrm{E}}=\left(2-k-\pi_{i}\right), \quad i \in \mathcal{I}, k \in\{0,1\}, b \in\{1, \ldots, B\}, \\
\left((1-k)\left(1-\pi_{i}\right)+1 / p_{b}\right) z_{i b k} \leq u_{i b k}^{\mathrm{S}}, \quad i \in \mathcal{I}, k \in\{0,1\}, b \in\{1, \ldots, B\}, \\
u_{i b k}^{\mathrm{S}} \leq\left(1-k \pi_{i}\right) z_{i b k}, \quad i \in \mathcal{I}, k \in\{0,1\}, b \in\{1, \ldots, B\}, \\
u_{i, b+B i}^{\mathrm{E}}, k-1, k \leq\left(1-k \pi_{i}-\frac{1}{p_{B}}\right) z_{i b k}, \quad i \in \mathcal{I}, k \in\{0,1\}, b \in\{1, \ldots, B\} . \\
(1-k)\left(1-\pi_{i}\right) z_{i b k} \leq u_{i, b+B_{i}+k-1, k}^{\mathrm{E}}, \quad i \in \mathcal{I}, k \in\{0,1\}, b \in\{1, \ldots, B\},
\end{gathered}
$$

Constraint (3.16) makes sure that each task starts in a bucket. Constraints (3.17)-(3.18) ensure that only one task can be performed at a given time. Constraints (3.19)-(3.23) make sure that the continuous and binary 
variable get the correct definitions. Release times and deadlines can be modelled by restricting the proper $z_{i b k}, u_{i b k}^{\mathrm{S}}$ and $u_{i b k}^{\mathrm{E}}$.

The bucket-indexed formulation is similar to the time-indexed formulation and theoretically at least as strong as the time-indexed formulation. This is proven in [7]. In [31], a bucket-indexed approach is implemented in an iterative framework, successively dividing the scheduling horizon into larger pieces, for a resource-constrained project scheduling problem.

\subsection{Order-based formulations}

The order-based formulations are centred around pair of tasks and assuring that such pairs do not overlap. We will introduce two formulations that both use a binary decision variable that indicate in which order each pair of tasks occur, and a continuous variable representing the starting time of each task. By connecting the order-variable for each pair of tasks to their start time variables via constraints, each pair of tasks can be ensured not to overlap. How this is done is the main difference between the two formulations.

Introduce, for each pair of tasks $i \in \mathcal{I}, j \in \mathcal{I}, j \neq i$, the binary variable

$$
y_{i j}= \begin{cases}1, & \text { if task } i \text { execute before task } j, \\ 0, & \text { otherwise. }\end{cases}
$$

For each task $i, i \in \mathcal{I}$, introduce the continuous variable

$$
s_{i}=\text { start time of task } i \text {. }
$$

\section{Disjunctive formulation}

The disjunctive formulation uses the previously defined variables to create a complete schedule for a single machine scheduling problem with release times and deadlines. The formulation is

$$
\begin{gathered}
s_{j} \geq s_{i}+p_{i}-M\left(1-y_{i j}\right), \quad i \in \mathcal{I}, \quad j \in \mathcal{I}, i \neq j, \\
y_{i j}+y_{j i}=1, \quad i \in \mathcal{I}, \quad j \in \mathcal{I}, i<j, \\
r_{i} \leq s_{i} \leq d_{i}-p_{i}, \quad i \in \mathcal{I},
\end{gathered}
$$

where Constraints (3.26)-(3.27) assure that no two tasks overlap, and Constraint (3.28) makes each task respect its release time and deadline. Note that Constraint (3.26) is of big-M type and will yield a weak LP-relaxation.

The first article to introduce an order-based decision variable for a scheduling problem was [23] in the context of job-shop scheduling. In the same year, a variant of defining an order-based variable was also studied in [24] for the travelling salesman problem. In this setting, the binary decision variable denoted if a task was the immediate predecessor to another task in a schedule or not. 
The polyhedral studies of disjunctive formulation for the single machine scheduling problem was started by [3], who studied the polyhedra in the context of job shop scheduling for a wide range of constraints. An extended description of the polyhedra of the disjunctive formulation was given a few years later in [27], who utilised the results in a cutting plane procedure.

\section{Linear-ordering formulation}

The linear-ordering formulation is a variation of the disjunctive formulation where the order-based decision variables are connected with the triangle inequality instead of big-M constraints. In order to get a well-defined schedule, an objective function to minimise the sum of the starting times is used. The formulation assumes that $\mathcal{I}$ is ordered with respect to ascending release times, and solving

$$
\min \sum_{i \in \mathcal{I}} s_{i}
$$

subject to

$$
\begin{gathered}
y_{i j}+y_{j i}=1, \quad i \in \mathcal{I}, j \in \mathcal{I}, i<j, \\
s_{j} \geq r_{i} y_{i j}+\sum_{\substack{k \in \mathcal{I}, k<i, k \neq j}} p_{k}\left(y_{i k}+y_{k j}-1\right)+\sum_{\substack{k \in \mathcal{I}, k \geq i, k \neq j}} p_{k} y_{k j}, \quad i \in \mathcal{I}, j \in \mathcal{I}, \\
s_{i} \leq d_{i}-p_{i}, \quad i \in \mathcal{I},
\end{gathered}
$$

will create a feasible schedule for the single machine scheduling problem with release times and deadlines. In the formulation, Constraints (3.30)-(3.31) assure a valid sequence, Constraint (3.32) forces each task respect its release time, while making sure that tasks does not start before preceding tasks are finished. Constraint (3.33) forces each task to finish before its deadline. Note that any feasible order given by the binary decisions $y_{i j}$ will have at least one corresponding feasible schedule; the one where all tasks are scheduled as early as possible with respect to their order.

In [12], Constraint (3.31) was first used as an extension to the disjunctive formulation when studying theoretical properties of formulations. But the linear ordering formulation, as shown above, was introduced in [25], along with some valid inequalities.

\subsection{Position-based formulation}

The position-based formulation is organised around the notion that a schedule can be represented by the position each task occupies. The formulation includes a binary decision variable that specify if a task is performed at a specific position, and a continuous variable for the starting time of the task processed at each position. 
Let the ordered set $\mathcal{P}$ contain a position for each task that shall be scheduled. For task $i \in \mathcal{I}$, and position $p \in \mathcal{P}$, introduce the binary variable

$$
z_{i p}= \begin{cases}1, & \text { if task } i \text { is performed in position } p \\ 0, & \text { otherwise. }\end{cases}
$$

For position $p \in \mathcal{P}$, introduce the continuous variable

$$
\mu_{p}=\text { start time of the task that execute in position } p \text {. }
$$

A solution to

$$
\begin{gathered}
\sum_{p \in \mathcal{P}} z_{i p}=1, \quad i \in \mathcal{I}, \\
\sum_{i \in \mathcal{I}} z_{i p}=1, \quad p \in \mathcal{P}, \\
\mu_{p}+\sum_{i \in \mathcal{I}} z_{i p} p_{i} \leq \mu_{p+1}, \quad p \in \mathcal{P}, \quad p<|\mathcal{P}|, \\
\sum_{i \in \mathcal{I}} z_{i p} r_{i} \leq \mu_{p} \leq \sum_{i \in \mathcal{I}} z_{i p}\left(d_{i}-p_{i}\right), \quad p \in \mathcal{P},
\end{gathered}
$$

will be a feasible schedule to the single machine scheduling problem with release times and deadlines. In the formulation, Constraint (3.36) assigns each task a position, Constraint (3.37) assigns each position a task, Constraint (3.38) ensures that two tasks at adjacent positions do not overlap, and Constraint (3.39) makes the start time of the task at each position respect its release time and deadline. Since there is no explicit connection between a task and its starting time, this formulation is a little less flexible dealing with time-related constraints, such as multiple time windows or minimal/maximal time lags, without introducing constructions like big-M constraints.

The notion of using a position-based variable in order to formulate an MIP for a scheduling problem was first introduced by [36] in context of machine scheduling. In [21], the polyhedra of the position-based formulation was studied. In recent years, the authors of [20] introduced additional inequalities for the position-based formulation based on the short processing time-rule. In the same year, [11] used the position-based formulation with some inequalities to explore neighbourhoods in a matheuristic.

\subsection{Comparisons of mixed integer programming formulations}

The MIP formulations introduced in the previous sections have been compared in different settings in the literature. There have been a few theoretical comparisons and primarily computational comparisons. This section attempts to summarise these results. 
In [12], the authors studied a series of linear programming relaxations originating from the disjunctive and time-based formulations. The authors showed that the linear programming relaxation of the time-based formulation dominates the linear programming relaxation of the disjunctive formulation for the problem of minimising the weighted sum of start times for single machine problem with release times $\left(1\left|r_{j}\right| \sum w_{j} S_{j}\right)$. A similar property is shown in [7], where the linear programming relaxation of the bucket-indexed formulation is shown to be at least as strong as the time-indexed formulation.

Four MIP formulations, the disjunctive, the time-indexed, the linear ordering, and the position-based formulations were compared in [19]. Test were conducted for the single machine scheduling problem when minimising the weighted completion time, the maximum lateness, the number of tardy jobs, and the total weighted tardiness. These comparisons were made both with and without release times, hence some of the problems were NP-complete and some can be solved in polynomial time. Their results indicated that the LPrelaxation of the disjunctive formulation and the position-based formulation were easier to solve then the time-indexed formulation and the linear ordering formulation, but they achieved weaker bounds. The position-based and the disjunctive formulations achieved a feasible solution the fastest even though the achieved solution was not always optimal.

A year later, computational comparisons between six MIP formulations were performed in [2]. The six formulations were the disjunctive, the timeindexed, the position-based, the linear-ordering and two additional formulations. The two additional formulations were a hybrid of disjunctive and position-based formulations, and a travelling salesman problem inspired formulation. The comparison was made for the single machine scheduling problem without release times while attempting to minimise total tardiness. Their results indicated that the position-based formulations performed best in reducing the total tardiness, and the hybrid version and the Travelling Salesman Problem (TSP) version performed worst.

When introducing the interval-indexed formulation in [4], the authors made a computational comparison. For the single machine scheduling problem with a generic objective function and release times $\left(1\left|r_{j}\right| \sum \alpha_{j} E_{j}+\beta_{j} T_{j}\right)$, the authors compared the interval-indexed formulation with the disjunctive formulation, and the time-indexed formulation. In this comparison, the timeindexed solved most instances to optimality followed by the interval-indexed formulation, and last was the disjunctive formulation. Worth to note is that the disjunctive formulation processed nodes in the MIP solver much faster than the time-indexed formulation on these instances. The authors also made a comparison on a problem without release times $\left(1|-| \sum w_{j} T_{j}\right)$. In this setting, they compared the interval-indexed formulation with the time-based formulation and the linear-order formulation. In this comparison, the interval-indexed approach performed best with respect to the relative MIP-gap, but when com- 
paring the number of instances solved to optimality, none of the formulations performed better than the others. 



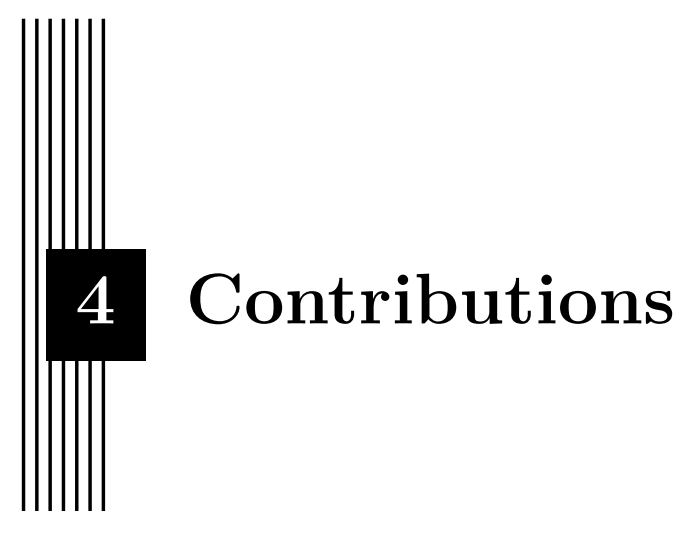

In this chapter, the main contributions of the appended articles are summarised. The focus of this thesis has been to develop a scheduling tool that uses optimisation methods for solving an avionic scheduling problem. The resulting method is matheuristic which at its core uses a constraint generation procedure.

The structure of this chapter is as follows. Section 4.1 describes the contributions from an avionics perspective. Section 4.2 gives an overview of the constraint generation procedure developed for the avionic scheduling problem addressed in this thesis. Section 4.3 views the constraint generation procedure in the light of a single machine scheduling problem. The purpose of this is to highlight the connections between the constraint generation procedure and scheduling. The last section, Section 4.4, describes the matheuristic that we have developed, in which the relaxed problem of the constraint generation procedure is solved using an ALNS.

\subsection{A large-scale problem in avionic scheduling}

This thesis addresses a scheduling problem proposed by our industrial partner Saab. This scheduling problem is of interest in the development of future IMA systems and it was introduced in Paper A.

In the sections that follow, different aspects of the contributions are described. The first section describes the motivation for the scheduling problem. The second section describes instances for the scheduling problem and gives 
some background of the public instances that have been constructed as part of the research. The last section gives a short description of the algorithmic components and overall design considerations of the scheduling tool.

\section{Problem background}

During the last decades, avionic systems have grown increasingly complex. One challenge that follows is that the schedulability analysis required for such systems also grows more challenging. In order to do a schedulability analysis of the IMA system described in Paper A, multiple aspects would need to be considered. Our focus is to produce a pre-runtime schedule that fulfils a set of predefined constraints or to show that no such schedule exists. A pre-runtime schedule that satisfy these constraints is necessary for the IMA system to work properly.

Two major challenges in this pre-runtime scheduling problem are the large number of tasks on the CMs and the interaction between CN and CM scheduling. In order to handle these challenges and solve the problem that is of interest for Saab, new methods need to be developed. This thesis makes contributions to avionic scheduling by presenting methods based on optimisation to solve a relevant pre-runtime scheduling problem.

\section{Problem instances}

A challenge with the work in this thesis has been test instances. We have been provided with relevant instances from Saab, but it has not been possible to make these available for other researchers.

For this reason, we have also created public instances. These instances are generated using a tool that has been developed in dialogue with Saab. The instances generated by this tool have the same characteristics as the instances provided by Saab but are generic enough to be publicly available. The public instances generated by this tool are by Saab considered industrially relevant for the development of future avionic systems. Results of this work can be seen in Paper C, where 120 instances divided into Categories A-D are introduced.

Our instances are a contribution to scheduling of avionic systems since there are few publicly available instances for large-scale pre-runtime scheduling problems.

\section{Developing a scheduling tool}

When constructing a scheduling tool, several things need to be considered. Since it is unknown if a particular instance has a feasible schedule or not, it is useful to have components in the scheduling tool that focus on proving infeasibility. 
At the core of the method implemented in this scheduling tool is a constraint generation procedure. Part of this procedure is to remove constraints and only add them if it is necessary. As a result of this, each step of the constraint generation procedure involves solving a relaxation of the original problem. If this relaxation is infeasible in any step, we have proved that the original problem is infeasible. Additionally, we have pre-processing components that can discover infeasibility with respect dependencies and fixed tasks. These pre-processing components are further described in Paper A.

Since an avionic system is developed iteratively, it is useful to make use of schedules or information from similar instances. The choice of using a constraint generation procedure has been done with this in mind. Since this procedure generates constraints, the constraints generated for an instance might be useful for another instance if there are minor differences between them.

\subsection{A solution approach for avionic scheduling}

The avionic scheduling problem proposed by Saab is challenging. In the beginning of the project we developed a standard MIP model suited for this type of scheduling problem. When we tried to solve this MIP model for the smallest instance provided by Saab, we failed to achieve a feasible solution within a week of runtime. This smallest instance had about 6500 tasks and we knew that the largest instances provided by Saab would have more than 40000 tasks. Thus, something needed to be done. This section presents the constraint generation procedure which is the core of the method that we have developed to solve such problems.

\section{A constraint generation procedure}

The scheduling problem consists of four categories of constraints: CM scheduling, AM scheduling, CN scheduling and Precedence relations constraints. The most challenging of these is the CM scheduling. This is primarily due to the many tasks on the CMs. However, we have learned that the instances provided by Saab have some characteristics that we can take advantage of. The most important of these are:

- There exist fixed tasks on CMs

- Many requirements of the problem are not difficult to fulfil in isolation

The constraint generation procedure that is described is suitable for these characteristics. The idea behind the constraint generation procedure is to make a relaxed scheduling decision, while respecting the other constraints of the problem. The relaxed scheduling decision is then the evaluated in a subproblem. This subproblem has been designed so that if a solution to the 
complete problem is not found during the evaluation, it is always possible to generate additional constraints for the relaxed scheduling decision.

\section{An assignment of sub-intervals to tasks}

In this section we will describe the relaxation that is made in the constraint generation procedure in further detail. Due to the fixed tasks, the CN task time requirement and pre-processing components, there exist natural subintervals for each task. In a feasible schedule, each task must be placed inside one of its sub-intervals. Instead of forcing tasks on the same module not to overlap, the relaxed problem creates an assignment of sub-intervals to tasks. This will ensure that on each module, no tasks will overlap fixed tasks. However, tasks that has been assigned to intersecting sub-intervals on the same module might still overlap. In order to make a good assignment, this assignment of sub-intervals to tasks is made with respect to the other constraints of the original problem along with a set of constraints that is a relaxation of the overlap requirement.

An assignment of sub-intervals to tasks that fulfil the described constraints is then evaluated in a subproblem. To this subproblem, soft overlap constraints are added and the objective aims to minimise overlap between tasks. If a subproblem does not find a feasible schedule to the original problem, we add overlap constraints that failed to be fulfilled in the subproblem, into the subsequent relaxed problems and subproblems. This will ensure a change in the next iteration of the constraint generation procedure. As constraints are added to the relaxed problem, the relaxed problem will converge to a complete model for the original problem. However, in practise we rely on not generating too many constraints. For further details of the constraint generation procedure in our scheduling tool, see Paper C.

\section{Problem formulation and integer programming}

In the appended papers, the relaxed problem and the subproblem of the constraint generation procedure are formulated and solved as MIP models. This section describes the progress with respect to the MIP models that is used in the constraint generation procedure.

Constructing efficient MIP models has been an important part of each appended paper. It is important since it is the foundation for both the exact solution approaches in Paper $\mathrm{A}-\mathrm{C}$ and the matheuristic of Paper C. During this work, the choice of MIP formulation has had a significant impact on the computational performance of the methods.

As this project has progressed, different technical aspects have been gradually introduced. In Paper A and Paper B, a restricted CN problem formulation was used where no co-allocation of messages was allowed. In Paper C, this 
restriction is removed, and co-allocation of messages is allowed, based upon the ideas in [32].

Another difference between the papers has been the relaxation used in the constraint generation procedure. In Paper A, each CM-task is assigned to be in one of the parts of the major period not occupied by fixed tasks. Each such part was referred to as a section. This was changed in Paper B, where the concept of sub-intervals was introduced. With the introduction of sub-intervals, a sub-interval was assigned to each task instead of assigning a section to each task. Assigning sub-intervals to tasks made the relaxed problem stronger but gives less flexibility in the subproblem compared to assigning sections to tasks.

However, an advantage of assigning sub-intervals to tasks is the strong connection to the requirements of $\mathrm{CN}$ task times. Especially for the tasks that are responsible for sending a message in a $\mathrm{CN}$ slot. As a result of this, the assignment of sub-intervals gives a complete CN schedule. In Paper B and Paper C, this means that for each subproblem, the CN schedule has already been determined by the solution from the preceding relaxed problem.

The MIP formulations for the relaxed problem and the subproblem are further improved in Paper C. In Paper C, the concept of sub-interval assignment is combined with the $\mathrm{CN}$ message co-allocation, see [32], one of the sequencing formulations tested in the Bachelor thesis [6], together with some further improvements.

\subsection{An exact solution approach for single machine scheduling}

This section aims at describing the connection between the constraint generation procedure used in the appended papers and MIP formulations for scheduling. Therefore, the constraint generation procedure is presented for the single machine scheduling problem with release times and deadlines, like the MIP formulations were in Chapter 3.

\section{Notation}

Let $\mathcal{I}$ denote the set of tasks to be scheduled. For each task $i \in \mathcal{I}$, let $\mathcal{Q}_{i}$ denote its set of sub-intervals and $p_{i}$ its duration. For each sub-interval $q \in \mathcal{Q}_{i}$ of task $i$, let $t_{i q}^{\mathrm{r}}$ and $t_{i q}^{\mathrm{d}}$ denote its start and end, respectively. Let $\mathcal{S}$ denote the set of sequences (set of tasks with overlapping sub-intervals) and $\mathcal{S}^{\text {gen }}$ the set of sequences generated by the subproblem. For a sequence $s \in \mathcal{S}$, let $\mathcal{I}_{s}^{\mathrm{O}}$ denote its set of tasks. Also, let $\mathcal{R}$ denote the set of segments. The segments are the set of partitions of the scheduling horizon obtained by $\left\{\left(t_{i q}^{\mathrm{r}}, t_{i^{\prime} q^{\prime}}^{\mathrm{d}}\right)\right.$ : $\left.t_{i q}^{\mathrm{r}}<t_{i^{\prime} q^{\prime}}^{\mathrm{d}}, q^{\prime} \in \mathcal{Q}_{i^{\prime}}, q \in \mathcal{Q}_{i}, i^{\prime} \in \mathcal{I}, i \in \mathcal{I}\right\}$. For a given segment $r \in \mathcal{R}$, let $\mathcal{Q}_{i r}$ 
denote the set of sub-intervals of task $i$ that is completely contained within segment $r$.

\section{Variables}

This section introduces the variables used for the relaxed problem and the subproblem that is described in the following sections. For task $i \in \mathcal{I}$, introduce the continuous variable

$$
x_{i}=\text { start time of task } i \text {. }
$$

For task $i \in \mathcal{I}$ and sub-interval $q \in \mathcal{Q}_{i}$, introduce the binary variable

$$
\alpha_{i q}= \begin{cases}1, & \text { if task } i \text { is assigned to sub-interval } q, \\ 0, & \text { otherwise. }\end{cases}
$$

For $i \in \mathcal{I}, i^{\prime} \in \mathcal{I}, i \neq i^{\prime}$, introduce the binary variable

$$
y_{i i^{\prime}}= \begin{cases}1, & \text { if task } i \text { starts before task } i^{\prime} \\ 0, & \text { if task } i^{\prime} \text { starts before task } i\end{cases}
$$

For each sequence $s \in \mathcal{S} \cup \mathcal{S}^{\text {gen }}$, introduce the binary variable

$$
\beta_{s}= \begin{cases}1, & \text { if there is no overlap between tasks in sequence } s \\ 0, & \text { otherwise }\end{cases}
$$

\section{Relaxed problem}

The purpose of the relaxed problem is to create an assignment of sub-intervals to tasks. Different objective functions can be used to guide this search, and in our work, the choice of objective function has had a great impact on the computational efficiency of the constraint generation procedure. For objective functions that were used to guide the search for our avionic scheduling problem, see Paper A and Paper C.

The relaxed problem for the single machine scheduling with release times and deadlines is formulated as

$$
\begin{gathered}
\sum_{q \in \mathcal{Q}_{i}} \alpha_{i q}=1, \quad i \in \mathcal{I}, \\
\sum_{q \in \mathcal{Q}_{i}} t_{i q}^{\mathrm{r}} \alpha_{i q} \leq x_{i} \leq \sum_{q \in \mathcal{Q}_{i}} t_{i q}^{\mathrm{d}} \alpha_{i q}-p_{i}, \quad i \in \mathcal{I}, \\
\sum_{i \in \mathcal{I}} \sum_{q \in \mathcal{Q}_{i r}} p_{i} \alpha_{i q} \leq \max _{i \in \mathcal{I}} \max _{q \in \mathcal{Q}_{i r}} t_{i q}^{\mathrm{d}}-\min _{i \in \mathcal{I}} \min _{q \in \mathcal{Q}_{i r}} t_{i q}^{\mathrm{r}}, \quad r \in \mathcal{R},
\end{gathered}
$$




$$
\begin{gathered}
y_{i i^{\prime}}+y_{i^{\prime} i}=1, \quad i \in \mathcal{I}_{s}, \quad i^{\prime} \in \mathcal{I}_{s}, i<i^{\prime}, \quad s \in \mathcal{S}^{\text {gen }}, \\
x_{i}+p_{i}-M\left(1-y_{i i^{\prime}}\right) \leq x_{i^{\prime}}, \quad i \in \mathcal{I}_{s}, i^{\prime} \in \mathcal{I}_{s}, \quad i \neq i^{\prime}, s \in \mathcal{S}^{\text {gen }} .
\end{gathered}
$$

Constraint (4.1) forces each task to be performed in one of its sub-intervals and Constraint (4.2) ensures that the release-time and deadline of this subinterval is respected. Constraint (4.3) assures that the capacities of the segments in the machine are not exceeded. This can be viewed as a relaxation of the constraints that forces the tasks on this machine not to overlap. Constraints (4.4)-(4.5) ensure that tasks of generated sequences do not overlap.

\section{Subproblem}

The responsibility of the subproblem is to evaluate a specific assignment of sub-intervals to tasks. In the subproblem, each task is restricted to be performed within the sub-interval given to it in the assignment that is evaluated. For each task $i \in \mathcal{I}$, let $\bar{q}_{i}$ denote the sub-interval it is assigned to. The assignment that is evaluated is typically a solution to the relaxed problem.

In this subproblem for the single machine scheduling problem with release times and deadlines, sequences of tasks are independent. However, this is a significant difference compared to the avionic scheduling problem. In the avionic scheduling problem, tasks in different sequences are not independent, for example due to precedence relations.

The subproblem minimises the number of sequences with tasks that do overlap and if necessary, utilise these sequences to create generated sequences. If a solution to the subproblem is obtained that has no tasks that overlap, a solution to the single machine scheduling problem with release times and deadlines has been found.

The subproblem is formulated as

$$
\min \sum_{s \in \mathcal{S}}\left(1-\beta_{s}\right)
$$

subject to

$$
\begin{gathered}
t_{i \bar{q}_{i}}^{\mathrm{r}} \leq x_{i} \leq t_{i \bar{q}_{i}}^{\mathrm{d}}-p_{i}, \quad i \in \mathcal{I}, \\
\beta_{s}=1, \quad s \in \mathcal{S}^{\mathrm{gen}}, \\
\beta_{s} \leq y_{i i^{\prime}}+y_{i^{\prime} i} \leq 1, \quad i \in \mathcal{I}_{s}, i^{\prime} \in \mathcal{I}_{s}, i<i^{\prime}, \quad s \in \mathcal{S} \cup \mathcal{S}^{\text {gen }}, \\
x_{i}+p_{i}-M\left(1-y_{i i^{\prime}}\right) \leq x_{i^{\prime}}, \quad i \in \mathcal{I}_{s}, i^{\prime} \in \mathcal{I}_{s}, i \neq i^{\prime}, s \in \mathcal{S} \cup \mathcal{S}^{\text {gen }},
\end{gathered}
$$

Constraint (4.7) restricts each task to be performed within its sub-interval. Constraint (4.8) ensures that tasks in previously generated sequences do not overlap. This ensure that any sequence that has tasks that fail to overlap is not in the set of generated sequences. Constraint (4.9) defines the values of the of the $\beta$-variables. Constraint (4.10) ensures that tasks do not overlap unless they are allowed to. Note that the interpretation of the variable $y_{i i^{\prime}}$ 
is slightly different in the subproblem compared to the relaxed problem in the cases the tasks are not forced to overlap.

\section{Relations to single machine scheduling formulations}

This section describes the relationship between the presented constraint generation procedure and the MIP formulations presented in Chapter 3. The constraint generation procedure described in the previous sections can be seen as a combination of two single machine scheduling formulations: the interval-indexed formulation and the disjunctive formulation. Since it uses sub-intervals, it is similar to the interval-indexed formulation with the difference that in this problem the sub-intervals of a task are disjoint. In this sense it is closer to the segmented version of the problem described in [9].

Decomposing the scheduling problem into a relaxed problem that creates an assignment of sub-intervals to tasks and a subproblem that evaluates such an assignment is done in [9]. However, the constraint generation procedure described in the previous sections and the one described in [9] differs in the feedback mechanism. In [9], an assignment of sub-intervals to tasks is forbidden in the relaxed problem using the original variables, but in our case, we add additional variables and sequencing constraints. The main reason for this is that the tasks of different sequences are not independent in the avionic scheduling problem. Furthermore, by adding variables and sequencing constraints as feedback, we can exit the subproblem without proving infeasibility, which is useful for the avionic scheduling problem.

\subsection{A matheuristic for avionic scheduling}

The computational bottleneck of the constraint generation procedure presented in the previous sections is the relaxed problem. When the instances become larger, it gets difficult for a MIP solver to find a feasible solution to the relaxed problem. In Paper C, we have developed a matheuristic based on the constraint generation procedure presented in the previous sections. The difference compared to the exact constraint generation procedure is that the matheuristic solves the relaxed problem using an ALNS. The ALNS solves a series of MIP models, instead of solving the relaxed problem in a single step. The reason to use an MIP model comes from the fact that the problem contains both binary and continuous variables.

A difficulty with the relaxed problem for larger instances is to achieve feasibility. In order to handle this, we do a reformulation of the relaxed problem. In this reformulation, instead of treating the constraints as hard, the constraints are treated as soft with a penalty if they are not feasible. A few constraints, the ones forcing the AMs to be scheduled and the ones forcing tasks to be placed within one of their sub-intervals, are kept as hard in the MIP model. A solution satisfying these hard constraints is a solution in our 
search. In order to create an initial solution, the matheuristic also includes a constructive heuristic that utilises MIP models, see Paper C.

At the centre of our ALNS is the assignment of sub-intervals to tasks. In each iteration, the ALNS makes two important decisions, which tasks that can be reassigned to new sub-intervals and whether to focus on achieving feasibility with respect to the relaxed problem or improving the relaxed problem objective. These decisions are used to construct a MIP model. This MIP model is then solved to get a complete solution to the reformulated relaxed problem.

An aspect that is important for the search is the trade-off between feasibility and the relaxed problem objective. In the ALNS, this is treated in a strategic oscillation fashion, where the search alternate between focusing on achieving feasibility or improving the relaxed problem objective.

With this matheuristic our scheduling tool has been able to solve larger instances compared to if the scheduling tool used the exact constraint generation procedure. The instance with most tasks that we solve in Paper $\mathrm{C}$ is one having 45988 tasks and $2011 \mathrm{CN}$ messages, and it was solved when the scheduling tool used the matheuristic. 



\section{Bibliography}

[1] J.M. van den Akker, C.P.M. van Hoesel, and M.W.P. Savelsbergh. "A polyhedral approach to single machine scheduling problems". In: Mathematical Programming 85.3 (1999), pp. 541-572.

[2] K.R. Baker and B. Keller. "Solving the single-machine sequencing problem using integer programming". In: Computers $\&$ Industrial Engineering 59.4 (2010), pp. 730-735.

[3] E. Balas. "On the facial structure of scheduling polyhedra". In: Mathematical Programming Study 24 (1985), pp. 179-218.

[4] P. Baptiste and R. Sadykov. "On Scheduling a Single Machine to Minimize a Piecewise Linear Objective Function: A Compact MIP Formulation". In: Naval Research Logistics 56.6 (2009), pp. 487-502.

[5] M. Blikstad, E. Karlsson, T. Lööw, and E. Rönnberg. "An Optimisation Approach for Pre-Runtime Scheduling of Tasks and Communication in an Integrated Modular Avionic System". In: Optimization and Engineering 19.4 (2018), pp. 977-1004.

[6] J. Boberg. "A comparison of sequencing formulations in a constraint generation procedure for avionics scheduling". LiTH-MAT-EX-2017/18SE. Bachelor Thesis. Linköping University, 2017.

[7] N. Boland, R. Clement, and H. Waterer. "A Bucket Indexed Formulation for Nonpreemptive Single Machine Scheduling Problems". In: Journal on Computing 28.1 (2016), pp. 14-30.

[8] E.H. Bowman. "The Schedule-Sequencing Problem". In: Operations Research 7.5 (1959), pp. 621-624. 
[9] E. Coban and J.N. Hooker. "Single-facility scheduling by logic-based Benders decomposition". In: Annals of operations research 210.1 (2013), pp. 245-272.

[10] T. Danielsson, A. Pettersson, A. Gripsborn, and J. Håkegård. Ethernet for avionics. Patent. EP 2583419. Apr. 2016.

[11] F. Della Croce, F. Salassa, and V. T'Kindt. "A hybrid heuristic approach for single machine scheduling with release times". In: Computers 83 Operations Research 45 (2014), pp. 7-11.

[12] M.E. Dyer and L.A. Wolsey. "Formulating the single machine sequencing problem with release dates as a mixed integer program". In: Discrete Applied Mathematics 26.2-3 (1990), pp. 255-270.

[13] R. Fuchsen. "IMA NextGen: A New Technology For The Scarlett Program". In: IEEE Aerospace and Electronic Systems Magazine 25.10 (2010), pp. 10-16.

[14] M.R. Garey and D.S. Johnson. "Two-Processor Scheduling with StartTimes and Deadlines". In: SIAM Journal on Computing 6.3 (1977), pp. 416-426.

[15] R.E. Gomory. "Outline of an Algorithm for Integer Solution to Linear Programs". In: Bulletin of American Mathematical Society 64.5 (1958), pp. 275-278.

[16] R.L. Graham, E.L. Lawler, J.K. Lenstra, and A.H.G. Rinnooy Kan. "Optimization and approximation in deterministic sequencing and scheduling: A survey". In: Annals of Discrete Mathematics 5.C (1979), pp. 287-326.

[17] E. Karlsson and E. Rönnberg. "Explicit Modelling of Multiple Intervals in a Constraint Generation Procedure for Multiprocessor Scheduling”. In: Operations Research Proceedings 2017. Ed. by N. Kliewer, J.F. Ehmke, and R. Borndörfer. Springer International Publishing, 2018, pp. $567-572$.

[18] E. Karlsson, E. Rönnberg, A. Stenberg, and H. Uppman. A matheuristic approach to large-scale avionic scheduling. Tech. rep. LiTH-MAT-R2019/02-SE. Department of Mathematics, 2019.

[19] A.B. Keha, K. Khowala, and J.W. Fowler. "Mixed integer programming formulations for single machine scheduling problems". In: Computers $\&$ Industrial Engineering 56.1 (2009), pp. 357-367.

[20] A. Kooli and M. Serairi. "A mixed integer programming approach for the single machine problem with unequal release dates". In: Computers E Operations Research 51 (2014), pp. 323-330. 
[21] J.B. Lasserre and M. Queyranne. "Generic Scheduling Polyhedra and a New Mixed-Integer Formulation for Single-Machine Scheduling". In: Proceedings of the 2nd International IPCO Conference. Ed. by E. Balas, G. Cornuéjols, and R. Kannan. 1992, pp. 136-149.

[22] J.Y-T. Leung. Handbook of Scheduling: Algorithms, Models, and Performance Analysis. Boca Raton: Chapman \& Hall/CRC, 2004.

[23] A.S. Manne. "On the Job-Shop Scheduling Problem". In: Operations Research 8.2 (1960), pp. 219-223.

[24] C.E. Miller, A.W. Tucker, and R.A. Zemlin. "Integer Programming Formulation of Traveling Salesman Problems". In: Journal of the ACM (JACM) 7.4 (1960), pp. 326-329.

[25] G.L. Nemhauser and M.W.P. Savelsbergh. "A Cutting Plane Algorithm for the Single Machine Scheduling Problem with Release Times". In: Combinatorial Optimization, NATO ASI Series F: Computer and System Sciences. Ed. by M. Akgül, H. Hamacher, and S. Tüfekçi. Vol. 82. 1992, pp. 63-83.

[26] A.A.B. Pritsker, L.J. Waiters, and P.M. Wolfe. "Multiproject Scheduling with Limited Resources: A Zero-One Programming Approach". In: Management Science 16.1 (1969), pp. 93-108.

[27] M. Queyranne. "Structure of a simple scheduling polyhedron". In: Mathematical Programming 58.1-3 (1993), pp. 263-285.

[28] M. Queyranne and A.S. Schulz. Polyhedral approaches to machine scheduling. Tech. rep. 1994, pp. 1-62.

[29] Radio Technical Commission for Aeronautics (RTCA). "Software Considerations in Airborne Systems and Equipment Certification, RTCA DO-178C". 2012.

[30] Radio Technical Commission for Aeronautics (RTCA). "Integrated Modular Avionics (IMA) Development Guidance and Certification Considerations, RTCA DO-297". 2005.

[31] M. Riedler, T. Jatschka, J. Maschler, and G.R. Raidl. "An iterative time-bucket refinement algorithm for a high-resolution resourceconstrained project scheduling problem". In: International Transactions in Operational Research (2017), pp. 1-41. DOI: 10.1111/itor. 12445.

[32] E. Rönnberg. "Co-allocation of Communication Messages in an Integrated Modular Avionic System". In: Operations Research Proceedings 2017. Ed. by N. Kliewer, J.F. Ehmke, and R. Borndörfer. Springer International Publishing, 2018, pp. 459-465.

[33] A. Al-Sheikh. "Resource allocation in hard real-time avionic systems. Scheduling and routing problems". PhD thesis. INSA de Toulouse, 2011. 
[34] J.P. Sousa and L.A. Wolsey. "A time indexed formulation of nonpreemptive single machine scheduling problems". In: Mathematical Programming 54.1-3 (1992), pp. 353-367.

[35] A.A.J. Syed. "Model-Based Design and Adaptive Scheduling of Distributed Real-Time Systems". PhD thesis. Technische Universität Kaiserslautern, 2018.

[36] H.M. Wagner. "An integer linear-programming model for machine scheduling". In: Naval research logistics quaterly 6.2 (1959), pp. 131140.

[37] H. Wang and W. Niu. "A Review of Key Technologies of the Distributed Integrated Modular Avionics System". In: International Journal of Wireless Information Networks 25 (2018), pp. 358-369. 


\section{Papers}

The papers associated with this thesis have been removed for copyright reasons. For more details about these see:

http://urn.kb.se/resolve?urn=urn:nbn:se:liu:diva-156695 


\section{FACULTY OF SCIENCE AND ENGINEERING}

Linköping Studies in Science and Technology, Licentiate Thesis No. 1844, 2019 Department of Mathematics

Linköping University

SE-581 83 Linköping, Sweden

www.liu.se 